\title{
Prevalence of Itching in Dengue and Its Correlation with Thrombocytopenia in Bundelkhand Region- A Tertiary Care Hospital-Based Study in the Region of Central India
}

\author{
Zaki Siddiqui ${ }^{1}$, Shyam Mohan Yadav², Kshitiz Nath³, Gopambuj Singh Rathor ${ }^{4}$ \\ ${ }^{1}$ Department of Internal Medicine, MLB Medical College, Jhansi, Uttar Pradesh, India. ${ }^{2}$ Department of Internal \\ Medicine, MLB Medical College, Jhansi, Uttar Pradesh, India. ${ }^{3}$ Department of Internal Medicine, MLB Medical College, \\ Jhansi, Uttar Pradesh, India. ${ }^{4}$ Department of Internal Medicine, MLB Medical College, Jhansi, Uttar Pradesh, India.
}

\section{ABSTRACT}

\section{BACKGROUND}

According to NVBDCP ${ }^{1}$ dengue is one of the vector born disease responsible for high morbidity as well as mortality in India. Previous studies have concluded that itching is common among dengue patients and could be a prognostic indicator. Our aim was to study the prevalence of itching as a symptom and its association with platelet count at presentation and discharge.

\section{METHODS}

This is a cross sectional study conducted among 73 patients of acute febrile illness in MLB Medical College, Jhansi, who were diagnosed to have dengue as per NVBDCP guidelines. We designed a proforma and collected data which was further analysed in detail.

\section{RESULTS}

Out of 73 patients, 15 patients (20.5\%) developed itching; mean platelet count was comparable at admission, but at the time of discharge mean platelet count was better in itching group when compared to non-itching group ( $p$ value 0.0085). Only 2 patients $(13.33 \%)$ among itching group required blood/platelet transfusion whereas 9 patients $(15.52 \%)$ among non-itching group required blood/platelet transfusion.

\section{CONCLUSIONS}

The prevalence of itching was significant in dengue patients and was associated with better rate of correction of thrombocytopenia and there was lesser need of platelet/blood transfusion among itching group.

\section{KEY WORDS}

Itching, Thrombocytopenia, Platelet Count, Platelet Transfusion
Corresponding Author: Shyam Mohan Yadav, Room No. 66, 80 PG Hostel, MLB Medical College, Jhansi, Uttar Pradesh, India. E-mail:mohshyam@icloud.com

DOI: $10.14260 / \mathrm{jemds} / 2020 / 226$

Financial or Other Competing Interests: None.

How to Cite This Article: Siddiqui Z, Yadav SM, Nath $K$, et al. Prevalence of itching in dengue and its correlation with thrombocytopenia in Bundelkhand region- a tertiary care hospital-based study in the region of Central India. J. Evolution Med. Dent. Sci. 2020;9(13):1050-1053, DOI: 10.14260/jemds/2020/226

Submission 20-01-2020, Peer Review 09-03-2020, Acceptance 16-03-2020, Published 30-03-2020. 


\section{BACKGROUND}

Skin manifestations can provide a clue to a treating physician enabling him to make a great decision for patient care in various systemic diseases which is an easy task and requires no extra effort ${ }^{2}$. This article focuses on co relation between itching as a symptomatic manifestation among dengue patents and its co relation with severity of thrombocytopenia at any time during the course of illness. Previous texts have mentioned that itching develops in significant no. of patient with dengue fever and according to WHO after critical phase of 24 to 48 hours recovery phase starts in patients which is attributed to next 48 to 72 hours which is due to reabsorption of interstitial fluid and patients may feel of wellbeing and their constitutional symptoms gets relieved, during this recovery phase they may start to develop rashes, pruritis, bradycardia and electrophysiological changes.

One important study was performed in Taiwan in 2016 by Hsin-Wei Huang ${ }^{1}$ et al and they co related the skin manifestation and thrombocytopenia as well as need of platelet/blood transfusion among dengue patients. forty five patients were included in the study and found that skin manifestations develops in significant number of patients during the course of illness are associated with better outcome and lesser need of blood transfusion and it could be taken as a prognostic marker however there is no study available which directly co-relates platelet count with itching and requirement of platelet/blood transfusion in patients who develops itching during the course of illness. They also found that swelling of palm and itching developed in significant no. of patient during the course of illness but their study was confined to co-relation of biochemical parameters and rash.

Another study was done by Saleem k et al on skin lesions in hospitalized cases of dengue fever and they found cutaneous manifestation were present in majority of patient and $68 \%$ patients developed skin lesion out of which $23 \%$ patients developed itching which again accounts for a significant proportion. There is no study performed exclusively on itching if it develops anytime during the course and its correlation with platelet counts and need of platelet/blood transfusion. Previous studies are suggestive of high prevalence of itching among dengue patients and there may be a role of itching in deciding the approach of treatment and it could be an important prognostic marker letting physician to have an advantage to avoid unnecessary investigation and to patients to avoid unnecessary blood transfusion/platelet transfusion so that to minimize the hazards of blood/platelet transfusion and unnecessary stay in hospital.

Dengue is a fast growing burden in most of the developing countries. According to $\mathrm{WHO}^{3}$ Dengue is the most rapidly spreading mosquito-borne viral disease in the world in the last 50 years incidence has increased 30 -fold with increasing geographic expansion to new countries and, in the present decade, from urban to rural settings. An estimated 50 million dengue infections occur annually and approximately 2.5 billion people live in dengue endemic countries. Cyclic epidemics are increasing in frequency and in-country geographic expansion is occurring in Bangladesh, India and Maldives - countries in the deciduous dry and wet climatic zone with multiple virus serotypes circulating. Reported case fatality rates for the region are approximately $1 \%$, but in India,
Indonesia and Myanmar, focal outbreaks away from the urban areas have reported case-fatality rates of $3-5 \% .^{3}$

According to $\mathrm{NVBDCP}^{1}$ dengue is one of the vectors born disease responsible for high morbidity as well as mortality in India. According to the data obtained 99913 cases was reported during the year 2015 out of which 220 death reported. Again 129166, 188401, 89974 cases were reported respectively in year 2016, 2017 and 2018 out of which 245,325 and 144 deaths have been reported.

Dengue may present in three different ways-

A. Classic Dengue Fever-

Symptoms are fever, chills, headache, retro-orbital pain, lumbosacral aching. A transient generalised erythematous rash may be present during first 24 to 48 hrs.

B. Dengue Haemorrhagic Fever (DHF)-

Most common with serotype 2 and mostly occurs during secondary infection and clinical spectrum includes fever lasting for 2 to 7 days along with haemorrhagic manifestation in form of at least one of the following-

- Petechiae, Purpura, Ecchymosis.

- $\quad$ Epistaxis, Gum Bleeding, Bleeding from any Site.

- Positive Tourniquet Test.

C. Dengue Shock Syndrome (DSS) (DHF + Shock)

Platelets become activated through binding of complement- and antibody-coated virus particles, by binding of DENV to DC-SIGN, and by direct binding of complement to the platelet surface. Activated platelets produce inflammatory cytokines and microparticles that act on vascular endothelium to promote plasma leakage.

A key clinical correlate of disease severity in dengue is thrombocytopenia. Platelet-count decline is temporally coincident with the critical phase of infection and the onset of vasculopathy and plasma leakage. Megakaryocytes are the main target for DENV in the bone marrow. Bone-marrow suppression, impaired thrombopoiesis, and increased peripheral platelet destruction are potential mechanisms that lead to dengue-associated thrombocytopenia. In addition, dengue thrombocytopenia has been correlated with complement activation and high levels of C3a and C5a. Antibody mediated platelet destruction, complementmediated platelet lysis and haemophagocytosis have all been proposed as mechanisms to drive thrombocytopenia. Various studies have mentioned skin manifestation in dengue fever. In a study in Taiwan by Hsin-Wei Huang et $\mathrm{al}^{5}$ concluded that skin manifestations are associated with lesser requirement of blood transfusion and can be a prognostic indicator.

\section{Categories of Skin Manifestation of Dengue}

1. Petechial lesion.

2. Morbilliform rash.

3. Maculopapular rash.

4. Only itching.

Itching is a common symptom of dengue fever and may appear during the recovery phase which starts on day $9^{\text {th }}$ of fever. 6 
The aim of our study was to determine the prevalence of itching as a symptom in dengue fever and its association with platelet count at the time of admission and discharge. This is first of its kind of study which correlates itching and disease outcome.

\section{METHODS}

This is a cross sectional study on 73 patients presented with acute febrile illness in the region of Bundelkhand, who got admitted to MLB medical college and diagnosed to have Dengue fever which was confirmed by ELISA method as per NVBDCP guidelines in microbiology dept. of MLB Medical College, Jhansi during the period of October 2018 to November 2018. The sample size was taken based on the convenience of the study. It was a cross sectional study and we divided them in two groups-

1. Itching group.

2. Non-itching group.

All patients went through thorough clinical evaluation and were further investigated for CBC, LFT, S. Creatinine and Urine analysis, KFT, X ray chest, USG whole abdomen. We studied itching as symptoms in all the patient admitted with dengue fever and categorised them into different groups using visual analogue scale. Cases were included on the basis of meeting the inclusion criteria. A proforma was designed for the collection of data and all the data obtained through it was further analysed in detail.

\section{Inclusion Criteria}

All patients admitted with dengue fever confirmed by ELISA as per NVBDCP guidelines at microbiology department of MLB medical college Jhansi.

\section{Exclusion Criteria}

1. Other diseases associated with thrombocytopenia such as B12 deficiency anaemia, aplastic anaemia.

2. Malarial pyrexia and other viral infection, sepsis with pancytopenia.

3. Drug allergy.

4. Known case of itching and urticarial.

All patients were managed as per NVBDCP guidelines and platelet transfusion was done as per the NVBDCP guidelines. ${ }^{7}$

\section{NVBDCP Guidelines}

1. Prophylactic platelet transfusion may be given at level of $<10,000 /$ cumm in absence of bleeding manifestations.

2. Platelet transfusion can be given in case of prolonged shock; with coagulopathy and abnormal coagulogram.

3. Platelet transfusion can be given in case of systemic massive bleeding in addition to red cell transfusion.

Patients were discharged on meeting the criteria for discharge of patients as per NVBDCP guidelines for dengue fever.

\section{Statistical Analysis}

The results were analyzed by using The Statistical Package for Social Science \{SPSS Version 20 will be used for Data Analysis.
Mean, median, and SD are used to describe quantitative data. Qualitative data are summarized using frequency and percentage.

\section{RESULTS}

We observed mean age of 26.47 years among all patients and out of 73 patients of males accounted for $64.59 \%$ (total no. 47) and females accounted for $35.61 \%$ (total no. 26). When we compared mean age for both the groups, we found $25.80 \pm 11.44$ years for itching group and $26.81 \pm 11$ for nonitching group and observed a p value of 1.01 which statistically non-significant. We also observed that 15 out of 73 patients developed itching which accounts for $20.5 \%$ of the total patients. Out of total 15 patients who developed itching as a symptom we further categorised them into different groups on the basis of severity of itching using visual analogue scale and found 6 patient developed itching of 3 to 5 score, 8 patient developed itching of 6 to 8 score patients and one patient developed itching of 9 to 10 score

Mean platelet count for 13 patients of itching group at the time of admission was 0.65 lacks as compared to 49 patients of non-itching group which was 0.69 lacks and when we compared both groups we observed a P value of 0.844 which was not significant and both groups were comparable at the time of admission on the basis of mean platelet count but at the time of discharge mean platelet count was 1.11 lacks for itching group and 0.82 lacks for non-itching group and on comparing both groups we observed a P value of 0.0085 which is statistically significant We also compared average hospital stay for both itching and non-itching group and found that average hospital stay was 4.7 days for itching group whereas it was 4 days for non-itching and on comparison between two groups we observed a $\mathrm{P}$ value of 0.143 which is statistically non-significant and was comparable between two groups.

We also found that blood/platelet transfusion was required in only 2 patients (13\%) in itching group whereas 9 (15.5\%) patients out of 49 patients required blood/platelet transfusion but since number patients were too small it needs to be studied further on a larger scale.

\begin{tabular}{|cccc|}
\hline Age Group (Years) & Total Cases & No. of Male Cases & No. of Female Cases \\
$18-24$ & 40 & 32 & 8 \\
$25-34$ & 20 & 9 & 11 \\
$35-44$ & 6 & 4 & 2 \\
45 and above & 7 & 2 & 5 \\
Total number & 73 & 47 & 26 \\
\hline \multicolumn{4}{r}{ Table 1. Demographic Distribution of Dengue } \\
\hline \multicolumn{4}{r}{}
\end{tabular}

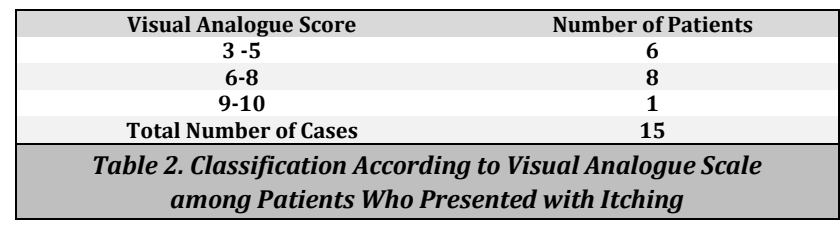

\begin{tabular}{|ccc|}
\hline \multicolumn{3}{|c|}{ Platelet Count in Itching and Non-Itching Group Who Did Not Require } \\
& Blood/Platelet Transfusion & \\
\hline Group & Mean Platelet Count at & Mean Platelet Count at \\
Itching & Presentation in Lacks & Discharge in Lacks \\
Non-itching group & 0.65 & 1.11 \\
P value & 0.69 & 0.82 \\
\hline Table 3. Mean Platelet Count at Admission and Discharge & 0.844 & $\mathbf{0 . 0 0 8 5}$ \\
\multicolumn{3}{|c|}{ among Itching and Non-Itching Group } \\
\hline
\end{tabular}




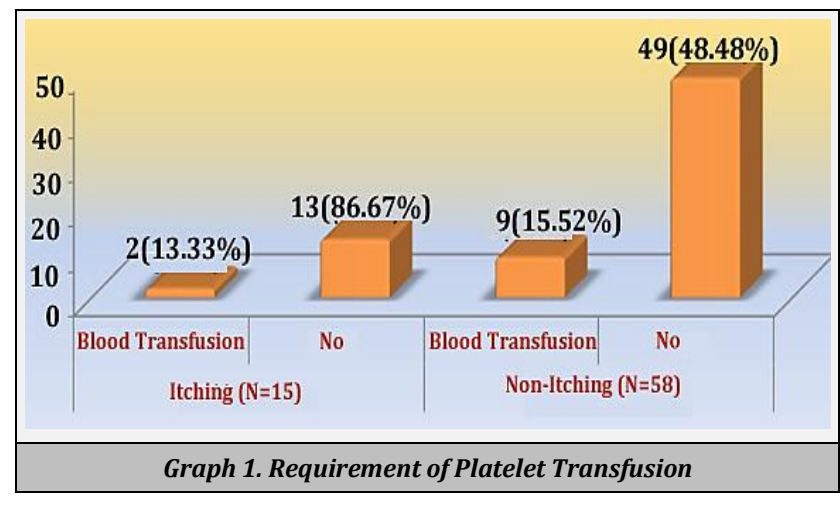

\section{DISCUSSION}

Dengue is a vector born disease caused by dengue virus (DENV) having a high prevalence rate in India and in Bundelkhand region responsible for significant morbidity and fear among population ${ }^{8}$. Most DENV infection are in apparent or cause mild symptoms however some patients present with severe symptoms characterised by vasculopathy and plasma leakage and sometimes death. Earlier studies have concluded a younger age and male predominance in the clinical spectrum of disease ${ }^{9}$ There are numerous studies conducted on clinical spectrum of the disease and one important study was conducted in Taiwan by Hsin-Wei Huang et.al in $2016^{5}$ which concluded that skin manifestation is associated with better clinical outcome but none of the studies correlate clinical outcome with itching.

A total 73 no. of in-patients were enrolled in our study during October 2018 to November 2018 out of which male to female ratio was 3:1 which support male predominance in DF as reported in dengue surveillance data from the Philippines for 2010 and from Singapore for 2009 and a study conducted by Deshwar R, Qureshi MI, Singh R in Department of medicine, military hospital Agra, Uttar Pradesh, 2015.10 Most of our patient belonged to the age group of 20-30 with mean age group of 26.47 which is suggestive of disease's trend towards younger population and on comparing both itching and nonitching group we observed a p value of 1.01 which is statistically insignificant and support the result of earlier study by Subhashisa Swain et al,11 on Distribution of and associated factors for dengue burden in the state of Odisha, India during 2010-2016 and study by Neelkanth S Patil et.al. ${ }^{12}$

We also found that among Dengue fever patients prevalence of itching was significant (20\%) supporting the previous study by Saleem K. et al, Journal of the College and Physicians and Surgeons, Pakistan where they found 23\% patients had generalised itching. ${ }^{13}$ We also found that rate of correction of thrombocytopenia was better among itching group ( $p$ value $=0.0085$ ) and there was lesser need of blood/platelet transfusion hence a better clinical outcome in dengue fever which has been previously reported by Hsin-Wei Huang et al. 5 This is first of its kind of study which correlates the itching as a symptom with platelet count and requirement of blood/platelet transfusion.

\section{CONCLUSIONS}

Prevalence of dengue fever is more in males and has a trend towards younger people. Itching develops in a significant number of patients and when it appears it signifies a good clinical outcome and correlates with better correction of thrombocytopenia and lesser requirement of blood/platelet transfusion than non-itching group. By looking at the clinical profile of patients we can conclude that itching is an important symptom and needs a better consideration and can be used as a prognostic marker.

\section{REFERENCES}

[1] NVBDCP current status in India.

[2] Huang HW, Tseng HC, Lee $\mathrm{CH}$, et al. Clinical significance of skin rash in dengue fever: a focus on discomfort, complications and disease outcome. Asian Pac J Trop Med 2016;9(7):713-8.

[3] World Health Organization. Dengue: Guidelines for diagnosis, treatment, prevention and control: new edition. Geneva: WHO, 2009.

[4] De Azeredo EL, Monteiro RQ, De-Oliveira LMP. Thrombocytopenia in Dengue: interrelationship between virus and the imbalance between coagulation and fibrinolysis and inflammatory mediators. Mediators Inflamm 2015;2015:313842.

[5] Huang HW, Tseng HC, Lee $\mathrm{CH}$, et al. Clinical significance of skin rash in dengue fever: a focus on discomfort, complications and disease outcome. Asian Pac J Trop Med 2016;9(7):713-8.

[6] Abbasi A, Butt N, Sheikh QH, et al. Clinical features, diagnostic techniques and management of dual dengue and malaria infection. J Coll Physicians Surg Pak 2009;19(1):25-9.

[7] Report NVBDCP, 2018 (nvbdcp.gov.in).

[8] Bhatt S, Gething PW, Brady OJ, et al. The global distribution and burden of dengue. Nature 2013;496(7446):504-7.

[9] Nascimento EJM, Hottz ED, Garcia-Bates TM, et al. Emerging concepts in dengue pathogenesis: interplay between plasmablasts, platelets and complement in triggering vasculopathy. Critical Reviews in Immunology 2014;34(3):227-40.

[10] Deshwal R, Qureshi MI, Singh R. Clinical and laboratory profile of dengue fever. J Assoc Physicians India 2015;63(12):30-2.

[11] Swain S, Bhatt M, Pati S, et al. Distribution of and associated factors for dengue burden in the state of Odisha, India during 2010-2016. Infect Dis Poverty 2019;8:31.

[12] Neelakanth SP, Neelam NR, Vikram L, et al. Study of hepatic involvement and thrombocytopenia in dengue. Journal Association Physicians India 2019;67(4):26-9.

[13] Saleem K, Shaikh I. Skin lesions in hospitalized cases of dengue fever. J Coll Physicians Surg Pak 2008;18(10):608-11. 